\title{
Pustular psoriasis of pregnancy: current perspectives
}

This article was published in the following Dove Press journal:

International Journal of Women's Health

\author{
Megha K Trivedi ${ }^{1,2}$ \\ Alexandra R Vaughn ${ }^{3}$ \\ Jenny E Murase ${ }^{1,4}$ \\ 'Department of Dermatology, \\ University of California, San \\ Francisco, CA, USA; ${ }^{2}$ Medical School, \\ University of Michigan, Ann Arbor, \\ MI, USA; ${ }^{3}$ College of Medicine, \\ Drexel University, Philadelphia, PA, \\ USA; ${ }^{4}$ Department of Dermatology, \\ Palo Alto Medical Foundation, \\ Palo Alto, CA, USA
}

\begin{abstract}
Pustular psoriasis of pregnancy (PPP) is a life-threatening condition for both the pregnant mother and fetus, and must be efficiently and accurately diagnosed and treated. This condition has historically been classified as a unique, separate dermatosis of pregnancy. However, current opinion and data suggest that it may be a variant of generalized pustular psoriasis. PPP typically occurs in the third trimester and is characterized by widespread coalescent pustules, desquamation, and systemic symptoms. Clinical features and histopathologic evaluation aid in diagnosis. Treatments during pregnancy include high-dose corticosteroids, cyclosporine, narrowband ultraviolet B radiation, infliximab, granulocyte and monocyte adsorptive apheresis, and systemic antibiotics. Both the mother and fetus should be closely monitored with appropriate laboratory studies for the duration of the pregnancy and postpartum.
\end{abstract}

Keywords: pustular psoriasis of pregnancy, impetigo herpetiformis, generalized pustular psoriasis, dermatoses of pregnancy

\section{Introduction}

\section{Background}

Pustular psoriasis of pregnancy (PPP) is a well-described dermatosis of pregnancy characterized by the fatal progression of disease for both mother and fetus if left untreated. The first reported cases of PPP appeared in the literature in the late nineteenth century. Historically, it has incorrectly been referred to as impetigo herpetiformis, but the disease is linked to neither bacterial colonization nor herpes simplex virus. ${ }^{1} \mathrm{PPP}$ is also referred to as generalized pustular psoriasis of pregnancy (GPPP), as this disease is thought to be a variant of generalized pustular psoriasis (GPP). ${ }^{1}$

Controversy exists about PPP's inclusion as a fifth dermatosis of pregnancy as it is more likely to be a variant of disease rather than a pregnancy-specific eruption. However, most experts agree on the classification of PPP as a dermatosis of pregnancy owing to the importance of early recognition and treatment. ${ }^{2}$

In brief, the clinical presentation of PPP includes the formation of sterile cutaneous pustules which tend to begin in the skin folds and spread centrifugally. This is typically accompanied by systemic changes including fever, neutrophilia, electrolyte abnormalities, and elevated inflammatory markers, among other findings. ${ }^{3}$

\section{Objective}

The objective of this review is to provide a comprehensive overview of PPP as well as a summary of the current medical consensus on the classification, pathogenesis, disease presentation, natural history, fetal and maternal outcomes, and most effective treatment for this disease. 


\section{Methods}

We conducted a review of the literature using the PubMed database, and supplemented the search with the Google Scholar database with the following search terms: "pustular psoriasis of pregnancy OR impetigo herpetiformis." The search was conducted from July 1, 2017 to July 30, 2017. This yielded 245 articles, case reports, and reviews, which were then further refined for inclusion during the development of this manuscript.

\section{Pathogenesis Classification controversy}

This disease was first described by von Hebra in a case publication which described five pregnant patients with systemic symptoms and pustular cutaneous eruption. Based on the clinical presentation, the author coined the term "impetigo herpetiformis." "However, in recent years, there has been increasing evidence to show that this condition is likely to be a variant of GPP which can flare owing to a variety of triggers, including metabolic disturbances, systemic steroid withdrawal, and pregnancy. The classification of PPP as a variant of disease or as a distinct disease state is still controversial and unclear. ${ }^{5,6}$ Since PPP is seen as a variant of GPP by many, a brief overview of the pathogenesis of GPP independent of pregnancy will be discussed here. This will then help to inform current treatment regimens for PPP.

\section{Immune-mediated and molecular pathogenesis}

GPP is typically grouped within the same category as other subtypes of psoriasis and is considered a disease of immune dysregulation. However, traditional treatments for plaque psoriasis are often ineffective for GPP, suggesting that certain molecular targets and pathways may differ or play a unique role in GPP compared to the more common types of psoriasis. A 2017 study conducted by Johnston and colleagues compared cytokine distributions in skin biopsy samples from GPP patients, plaque psoriasis patients, and healthy controls. Although significant overlap was seen between GPP and plaque psoriasis, their results emphasized the role of IL-1 and IL-36 in pustular disease. These interleukins are important in sustained neutrophil chemotaxis, which is responsible for the pustule formation. The authors also noted that keratinocytes, neutrophils, and monocytes were the most active cell types in GPP. Tumor necrosis factor- $\alpha$ (TNF- $\alpha$ ) and IL-17 $\alpha$ were also mentioned as being important in GPP pathogenesis, as they are in other psoriasis variants. ${ }^{7}$
Of note, one 1997 study reported low anti-elastase activities in the skin of impetigo herpetiformis patients. The authors described this disturbance in elastase activity inhibition as a major contributor to pustule formation in PPP (impetigo herpetiformis) patients. They noted that this explanation could also apply to GPP patients, further supporting the idea of PPP being a GPP variant. ${ }^{8}$

\section{Genetic studies}

There have also been several reports of genetic mutations predisposing patients to developing GPP. A report published in 2011 in which the authors performed exome sequencing of five unrelated individuals, all with a diagnosis of GPP, demonstrated mutations in an antagonist to IL-1 family receptors (IL36RN). IL36RN mutations were seen in three of the five patients who underwent sequencing. The authors noted that these findings supported a greater role of IL-1 and IL-36 in GPP pathogenesis. ${ }^{9}$ There have been several reports of this gene mutation in patients developing GPP or other pustular cutaneous conditions. There have also been reports of certain IL36RN mutations being more prevalent or associated with more severe forms of disease. There are a total of 17 mutations currently associated with GPP reported in the literature. ${ }^{10}$ Several reports from Asia describe the c. $115+6 \mathrm{~T}>\mathrm{C}$ mutation in the gene $I L 36 R N$ in patients presenting with GPP, suggesting that this may be a common mutation overall or in this subset of patients. ${ }^{11-13}$ To date, no known environmental factors have been solely implicated in the pathogenesis of PPP. However, environmental triggers seen to trigger GPP are also seen as common triggers for PPP. ${ }^{13}$ These triggers may induce PPP in patients with the genetic predispositions mentioned in this subsection.

\section{Clinical presentation of disease}

PPP tends to occur in the early part of the third trimester of pregnancy. This condition mostly resolves after parturition. However, the likelihood of recurrence in subsequent pregnancies is significant. ${ }^{14}$ There are reports in the literature of recurrence of disease in nine consecutive pregnancies for one patient. Oral contraceptives and menstrual cycle changes have also been associated with flares of PPP. ${ }^{15}$

Usually, PPP initially presents with pustules studded on erythematous patches within intertriginous areas, such as the axillae and skin folds of the breasts (Figure 1). Within one day, the pustules coalesce to form large dry plaques, which desquamate in large sheets episodically. The disease tends to spread centrifugally to the extremities, while sparing the face, palms, and soles. ${ }^{16}$ 


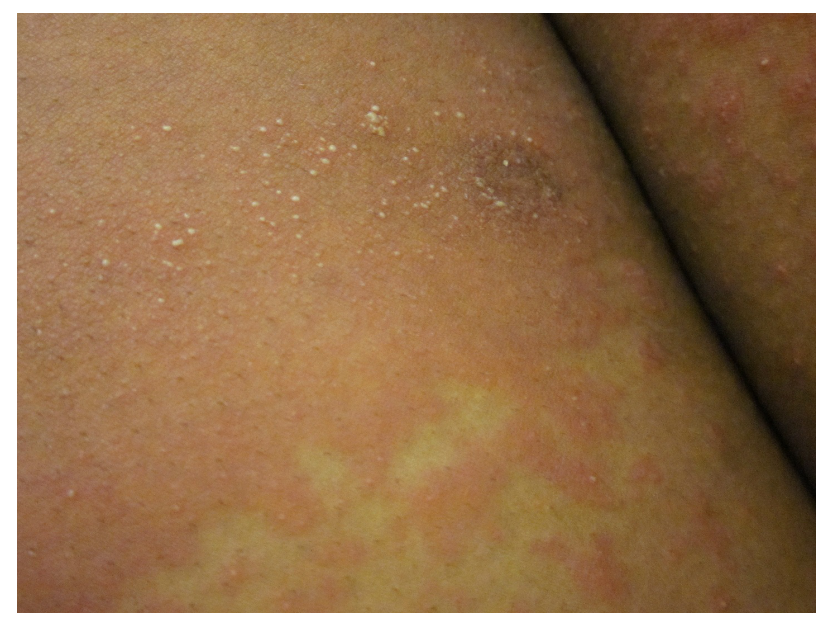

Figure I Micropustules on an erythematous plaque base. Note: Courtesy of Dr Darshan Vaidya and Dr George Kroumpouzos.

Unlike many other dermatoses of pregnancy, which are usually limited to the skin, PPP is frequently accompanied by systemic signs and symptoms of fatigue, fever, diarrhea, delirium, elevated markers of inflammation such as an increased erythrocyte sedimentation rate, and increased white blood cell counts. ${ }^{5,17}$ In severe cases, the rash of PPP can progress to erythroderma with resulting dangerous fluid and electrolyte imbalances, loss of thermoregulation in the skin, and the risk of secondary infection and sepsis. ${ }^{18}$

\section{Variations in PPP clinical presentation}

Although PPP often presents in the last trimester of pregnancy, there have been cases of PPP occurring in the postpartum period. There are a total of five reported cases of postpartum PPP in the literature. ${ }^{19-23}$ One of the most recent cases, published by Vaidya and colleagues, describes PPP eruption in a 27-year-old woman on postpartum day 1 (third pregnancy) after tubal ligation surgery. Her symptoms resolved after oral prednisone taper (initiated at $40 \mathrm{mg} /$ day). The patient had a history of PPP in her first pregnancy and an uneventful second pregnancy. ${ }^{23}$ The majority of the reported cases of postpartum PPP describe the onset of symptoms within 3-5 days of parturition. Most of the patients reported in these cases also had a history of gestational PPP in one or more pregnancies. All cases of postpartum PPP resolved with the initiation of oral corticosteroid treatment, similarly to cases of gestational PPP.

In addition to the typical pustular rash with associated systemic symptoms, PPP can present with other cutaneous manifestations and variations. A case report published in 2016 describes a 28-year-old patient with PPP who simultaneously developed acrodermatitis continua of Hallopeau
$(\mathrm{ACH})$, a variant of GPP characterized by pustules, erythema, and scale on the distal digits of both hands and feet. This condition is also associated with paronychia, anonychia, dystrophic nail changes, and skin atrophy. $\mathrm{ACH}$ is a very rare variant of GPP and this is the only reported case of $\mathrm{ACH}$ occurring concurrently with PPP. ${ }^{14}$

Another case of PPP published in 2001 described a 25-year-old female patient with unusual presentation of the disease. This patient developed lesions of PPP earlier than usual, at 16 weeks' gestation, and had significant involvement of her face, palms, and soles, areas typically spared in PPP. Although the termination of pregnancy or delivery of the baby often results in resolution of symptoms, this patient experienced worsening of her lesions as well as formation of large bullae and pitting edema after the termination of her pregnancy. The development of skin bullae was also an unusual presentation of the disease. ${ }^{24}$

PPP may also be confused with acute generalized exanthematous pustulosis (AGEP), which has a similar clinical presentation. A case published in 2009 describes a 23-yearold pregnant woman presenting with diffusely scattered pustules within plaques over the entirety of her body and histology showing perivascular lymphocytic infiltrate, dermal edema, and eosinophils, but no papillomatosis of the epidermis or acanthosis. This patient's clinical presentation of hundreds of non-follicular pustules on erythematous bases over her body and eosinophilic infiltrate on biopsy also led the authors to consider AGEP, which often presents similarly to PPP. AGEP, however, is almost always a drug-induced phenomenon and the agents that have been implicated in pregnancy-related AGEP in the literature include paracetamol, antimicrobials, and ingestion of spoiled pork. The patient did not have a history of such exposure and her symptoms resolved after the induction of labor and administration of corticosteroids. ${ }^{25}$

\section{Natural history of disease and fetal outcomes \\ Onset, progression, and recurrence}

In a large number of women with PPP, there is a positive personal or family history of psoriasis; nevertheless, there are still many cases without a known family history of psoriasis. ${ }^{17}$ Hypoparathyroidism, hypocalcemia, stress, and infections have been suggested as potential triggers of PPP, although causal associations have not been elucidated. ${ }^{16,18}$ In addition, if a woman develops PPP during one pregnancy, there is an increased risk of more severe outbreaks in subsequent pregnancies that may present earlier. 
Aggressive treatment and close monitoring of the mother and fetus are vital in the management of women with PPP. The more long-standing and severe the symptoms, the greater risk that PPP will cause placental insufficiency and harm the fetus, even resulting in stillbirth. ${ }^{26}$ Usually, symptoms of PPP will spontaneously regress upon delivery, which makes it easier to taper medications.

\section{Fetal distress and fetal outcomes in pustular psoriasis}

PPP can have significant effects on fetal health, leading to intrauterine growth restriction, and even miscarriage and stillbirth. When a pregnant woman has systemic signs and symptoms associated with PPP, the fetus must be closely monitored owing to the increased risk of placental insufficiency, fetal anomalies, and fetal demise. ${ }^{27}$

\section{Treatment}

PPP is one of the most serious, and potentially life-threatening dermatoses during pregnancy; it is also notoriously difficult to treat. ${ }^{28}$ Most importantly, early recognition and treatment of PPP are vital to ensure the best possible prognosis for both the mother and the fetus. ${ }^{3}$ In mild or early cases, symptoms of PPP can usually be adequately controlled with less than $30 \mathrm{mg} /$ day of systemic corticosteroids. However, more severe cases may necessitate higher doses of corticosteroids and some cases may be refractory to corticosteroids altogether. Other therapies, including cyclosporine, antibiotics, psoralen with ultraviolet A (PUVA), and systemic retinoids, have been used in reported cases. ${ }^{29}$

\section{Corticosteroids}

The treatment of PPP is crucial and should be initiated promptly to avoid serious complications in both mother and fetus. The guidelines for treatment of this condition have evolved over the past decade and several therapeutic options are now available for immunosuppression, which is the mainstay of treatment. Mild cases of PPP can be treated with lower dose corticosteroids, ranging from $15-30 \mathrm{mg} / \mathrm{day}$. Initial first-line treatment for severe PPP often consists of a trial of prednisone or prednisolone with doses ranging from $30 \mathrm{mg} /$ day to as high as $60-80 \mathrm{mg} /$ day. However, caution should be taken at higher doses as these can lead to reduced fetal reactivity on fetal monitoring. ${ }^{30}$ Most patients must continue oral corticosteroid therapy until at least parturition with a very gradual tapering of medication, given that PPP is likely to flare. . $^{2,31}$

\section{Cyclosporine}

Cyclosporine has historically been reserved for severe or recalcitrant cases of PPP. However, in 2012, the medical board of the National Psoriasis Foundation labeled cyclosporine as an appropriate first-line therapy for PPP. ${ }^{32}$ There have been several published cases reporting the successful use of cyclosporine in pregnant patients presenting with this condition. Cyclosporine is labeled as pregnancy category "C," but no adverse fetal outcomes related to medication use are reported in the literature. ${ }^{17,18,26,28,33-35}$ (Note that pregnancy categories are given according to the old US Food and Drug Administration pregnancy risk category lettering system; the new pregnancy and lactation labeling rules have yet to be applied to all medications. Therefore, medications are described with letters for context in this review.) The standard dose of cyclosporine for use in PPP patients is $2-3 \mathrm{mg} / \mathrm{kg}$ body weight/day, as this dose has been shown to be safe in pregnant transplant patients. ${ }^{2}$ Japanese guidelines also recommend the use of $3 \mathrm{mg} / \mathrm{kg}$ body weight/day for PPP patients. ${ }^{36}$ Cyclosporine, although thought to be safe to use in pregnancy, should not be used while breastfeeding as the amount of exposure to the infant through breastmilk is unclear. Patients should also be monitored with regular blood pressure readings and metabolic panels to assess renal function, as hypertension and renal impairment are potential adverse effects. ${ }^{17}$ Cyclosporine has traditionally been used as monotherapy or combination therapy with corticosteroids for recalcitrant cases of PPP. However, the medication has gained status as a first-line therapy more frequently for severe cases of PPP in recent years.

\section{TNF- $\alpha$ inhibitors}

There are only a few reports of TNF- $\alpha$ inhibitor (primarily infliximab) use for the treatment of PPP. Infliximab has been successfully used to treat the severe form of GPP, von Zombusch GPP, but it has also been implicated as an inducer of pustular psoriasis. ${ }^{37}$ Drugs such as infliximab, along with other TNF- $\alpha$ antibodies, are pregnancy category B, but there are limited controlled human data on their safety in pregnancy. A study involving first-trimester infliximab infusions for Crohn's disease and rheumatoid arthritis patients reported an overall normal distribution of pregnancy outcomes in the patient cohort. ${ }^{38}$ Theoretically, there is greater immunoglobulin $\mathrm{G}$ antibody placental transfer during the third trimester. Therefore, it has been recommended to limit infliximab use to the first 30 weeks of pregnancy. ${ }^{39}$ There are two reported cases of successful infliximab therapy for PPP treatment. One case involved a 
Japanese patient with a known IL36RN mutation who had failed granulocyte and monocyte adsorptive apheresis (GMA or GCAP) and oral prednisolone therapy. She was started on infliximab therapy at $5 \mathrm{mg} / \mathrm{kg}$ body weight at 22 weeks' gestation and stopped forming new skin lesions after her first infusion cycle. However, infusions were stopped after she entered her third trimester, and an emergency cesarean had to be performed owing to exacerbation of her condition. ${ }^{40}$ The second case involved a patient who had persistent rash and symptoms 7 weeks postpartum and was unable to taper her medications. Her symptoms cleared after three $5 \mathrm{mg} / \mathrm{kg}$ infusions given over 6 weeks. ${ }^{37}$

\section{Other treatments and considerations}

Light therapy including both narrow-band ultraviolet $\mathrm{B}$ (Nb-UVB) and PUVA, antibiotics, and GMA have been used to treat PPP. Nb-UVB is considered safe to use during pregnancy, as opposed to PUVA, which is traditionally reserved for the postpartum period. $\mathrm{Nb}-\mathrm{UVB}$ in combination with oral corticosteroids was effective in clearing recurrent PPP in a mother during her second pregnancy. Her symptoms were controlled with a regimen of $60 \mathrm{mg} /$ day prednisone and adjuvant $\mathrm{Nb}-\mathrm{UVB}$ therapy, which was added owing to inadequate control with steroids alone. She delivered a healthy baby at 38 weeks without complications. ${ }^{31}$ GMA has also been used for the treatment of PPP, although traditionally it has been used in the treatment of inflammatory bowel disease. The therapy is thought to exert immunosuppressive effects through the suppression of TNF- $\alpha$ and IL- $1 \beta$ production by monocytes. A case of PPP published in 2017, in which a patient experienced a PPP outbreak in the first trimester of her fourth pregnancy, reported successful GMA therapy. Therapy was continued throughout pregnancy with the patient undergoing a total of 14 cycles of treatment, during which time fetal intrauterine growth retardation improved and a healthy baby was born at the end of a 34 week gestation. ${ }^{41}$

Some experts recommend the use of antibiotic therapy concurrently with other therapies, even though the pustules of PPP are sterile. The antibiotic of choice is often a cephalosporin. ${ }^{42}$ As PPP is often associated with systemic and laboratory abnormalities, electrolytes, calcium, vitamin D, and albumin levels should be closely monitored and supplemented as needed. Hypocalcemia, in particular, should be assessed and managed as these patients have a higher risk of seizure. ${ }^{43}$ Lastly, as this condition generally improves after delivery, in severe or recalcitrant cases and/or when the health of the mother is compromised, induction of labor can be used as a therapeutic approach. ${ }^{43}$

\section{Postpartum considerations}

As mentioned in the subsection "Variations in PPP clinical presentation", PPP can often present or flare even during the postpartum period. However, these instances are often easier to manage as potential therapeutic regimens are less restricted. All therapies mentioned in this section can be safely used in the postpartum period. Special care must be taken for cyclosporine when breastfeeding as the effects and exposure of this medication through breast milk are unclear. ${ }^{17}$ Both methotrexate and oral retinoids are pregnancy category $\mathrm{X}$ and may result in severe fetal malformations, complications, and spontaneous abortion if used during pregnancy. However, these medications have been successfully used in recalcitrant cases of PPP occurring or flaring in the postpartum period. There have been two cases in the past decade reporting successful use of methotrexate for persistent PPP after delivery. ${ }^{44,45}$ One of the cases reports complete clearance of lesions after 8 weeks of therapy with methotrexate at $20 \mathrm{mg} /$ week. $^{45}$ Acitretin has also been used to successfully treat PPP in the postpartum period. A case describing a severe flare of PPP 10 days postpartum reports the use of acitretin initiated at a dose of $60 \mathrm{mg} /$ day in combination with cephalosporin. The patient was noted to have compete resolution of symptoms 3 months after delivery. However, breastfeeding while taking acitretin is not advised, since, similarly to isotretinoin, it is lipid soluble and there is a risk of high concentrations of medication in breast milk and subsequent retinoid toxicity to the fetus. ${ }^{46}$

\section{Conclusion}

Treatment of pregnancy-related dermatoses has been historically challenging as both maternal health and fetal compromise have to be adequately addressed when initiating therapy. Furthermore, PPP presents a unique challenge as this is a relatively rare diagnosis which many experts believe is a variant of GPP. However, it is crucial to identify and treat this condition as promptly as possible as it can result in a grim prognosis for both the mother and fetus if not recognized and treated. Biopsy as well as accurate clinical description are important diagnostic considerations as this condition can present similarly to other pustular diseases. Patients frequently tend to have recurrence of disease, which is often more severe and requires step-up or combination therapy. Genetic and pathogenesis studies have revealed significant involvement of IL-1 and IL-36 immune pathways in GPP. These findings may be helpful in developing future therapeutic modalities for both GPP and PPP. Current treatment regimens include high-dose systemic corticosteroids, 
cyclosporine, Nb-UVB, infliximab, GMA, antibiotic therapy and close systemic monitoring during pregnancy, and methotrexate, PUVA, and oral retinoids postpartum.

\section{Acknowledgment}

The authors thank Dr Darshan Vaidya and Dr George Kroumpouzos for providing the photograph for this publication.

\section{Disclosure}

The authors report no conflicts of interest in this work.

\section{References}

1. Danesh M, Pomeranz MK, McMeniman E, Murase JE. Dermatoses of pregnancy: nomenclature, misnomers, and myths. Clin Dermatol. 2016;34(3):314-319.

2. Lehrhoff S, Pomeranz MK. Specific dermatoses of pregnancy and their treatment. Dermatol Ther. 2013;26(4):274-284.

3. Gao Q-Q, Xi M-R, Yao Q. Impetigo herpetiformis during pregnancy: a case report and literature review. Dermatology. 2013;226(1):35-40.

4. Von Hebra F. Ueber einzelne während Schwangerschaft, des Wochenbettes und bei Uterinalkrankheiten der Frauen $\mathrm{zu}$ beobach-tende Hautkrankheiten. [Regarding individual skin diseases observed during pregnancy, the puerperium and in uterine diseases of women]. Wien Med Wochenschr. 1872;22:1197-1202.

5. Oumeish OY, Farraj SE, Bataineh AS. Some aspects of impetigo herpetiformis. Arch Dermatol. 1982;118(2):103-105.

6. Breier-Maly J, Ortel B, Breier F, Schmidt JB, Hönigsmann H. Generalized pustular psoriasis of pregnancy (impetigo herpetiformis). Dermatology. 1999;198(1):61-64.

7. Johnston A, Xing X, Wolterink L, et al. IL-1 and IL-36 are dominant cytokines in generalized pustular psoriasis. J Allergy Clin Immunol. 2017; 140(1):109-120.

8. Kuijpers ALA, Schalkwijk J, Rulo HFC, Peperkamp JJA, van de Kerkhof PCM, de Jong EMGJ. Extremely low levels of epidermal skin-derived antileucoproteinase/elafin in a patient with impetigo herpetiformis. Br J Dermatol. 1997;137(1):123-129.

9. Onoufriadis A, Simpson MA, Pink AE, et al. Mutations in IL36RN/ IL1F5 are associated with the severe episodic inflammatory skin disease known as generalized pustular psoriasis. Am J Hum Genet. 2011;89(3):432-437.

10. Takeichi T, Togawa Y, Okuno Y, et al. A newly revealed IL36RN mutation in sibling cases complements our IL36RN mutation statistics for generalized pustular psoriasis. J Dermatol Sci. 2017;85(1):58-60.

11. Wang T-S, Chiu H-Y, Hong J-B, Chan C-C, Lin S-J, Tsai T-F. Correlation of IL36RN mutation with different clinical features of pustular psoriasis in Chinese patients. Arch Dermatol Res. 2016;308(1):55-63.

12. Shiratori T, Fukai K, Yasumizu M, et al. IL36RN gene analysis of two Japanese patients with generalized pustular psoriasis. Int J Dermatol. 2015;54(2):e60-e62.

13. Sugiura K, Nakasuka A, Kono H, Kono M, Akiyama M. Impetigo herpetiformis with IL36RN mutations in a Chinese patient: a founder haplotype of c.115+6T >C in East Asia. J Dermatol Sci. 2015;79(3):319-320.

14. Shah A, Makhecha M. Pustular psoriasis of pregnancy with acrodermatitis continua of Hallopeau. Indian J Dermatol. 2016;61(1):123.

15. Gligora M, Kolavio Z. Hormonal treatment of impetigo herpetiformis. Br J Dermatol. 1982;107(2):253.

16. Razvi F, Haneef NS, Teja VS, Bang R, Hasnath FU. Generalized pustular psoriasis of pregnancy. J Med Allied Sci. 2015;5(2):51-53.

17. Flynn A, Burke N, Byrne B, Gleeson N, Wynne B, Barnes L. Two case reports of generalized pustular psoriasis of pregnancy: different outcomes. Obstet Med. 2016;9(2):55-59.
18. Shaw CJ, Wu P, Sriemevan A. First trimester impetigo herpetiformis in multiparous female successfully treated with oral cyclosporine. BMJ Case Rep. 2011;2011:bcr0220113915.

19. Ross MG, Tucker DC, Hayashi RH. Impetigo herpetiformis as a cause of postpartum fever. Obstet Gynecol. 1984;64(3S):49S-51S.

20. Abut YC, Bay B, Aldemir T. Postpartum impetigo herpetiformis complicated with hypovolaemic shock. Eur J Anaesthesiol EJA. 2009; 26(5):441-443.

21. Fouda UM, Fouda RM, Ammar HM, Salem M, Darouti ME. Impetigo herpetiformis during the puerperium triggered by secondary hypoparathyroidism: a case report. Cases J. 2009;2(1):9338.

22. Park HS, Youn SJ, Sung HT, Kim WS, Lee ES. A case of impetigo herpetiformis occurring in puerperium. Korean J Dermatol. 2004;42(6): 758-761.

23. Vaidya DC, Kroumpouzos G, Bercovitch L. Recurrent postpartum impetigo herpetiformis presenting after a "skip" pregnancy. Acta Derm Venereol. 2013;93(1):102-103.

24. Chang S-E, Cho S-Y, Bae J-Y, et al. A case of impetigo herpetiformis with unusual clinical features. J Dermatol. 2001;28(6):335-337.

25. Green MG, Bragg J, Rosenman KS, Keltz Pomeranz M. Pustular psoriasis of pregnancy in a patient whose dermatosis showed features of acute generalized exanthematous pustulosis. Int J Dermatol. 2009; 48(3):299-303.

26. Hazarika D. Generalized pustular psoriasis of pregnancy successfully treated with cyclosporine. Indian J Dermatol Venereol Leprol. 2009; 75(6):638.

27. Oumeish OY, Parish JL. Impetigo herpetiformis. Clin Dermatol. 2006; 24(2):101-104.

28. Luan L, Han S, Zhang Z, Liu X. Personal treatment experience for severe generalized pustular psoriasis of pregnancy: two case reports. Dermatol Ther. 2014;27(3):174-177.

29. Huang YH, Chen YP, Liang CC, Chang YL, Hsieh CC. Impetigo herpetiformis with gestational hypertension: a case report and literature review. Dermatology. 2011;222(3):221-224.

30. Ulubay M, Keskin U, Fidan U, et al. Case report of a rare dermatosis in pregnancy: impetigo herpetiformis. J Obstet Gynaecol Res. 2015; 41(2):301-303.

31. Bozdag K, Ozturk S, Ermete M. A case of recurrent impetigo herpetiformis treated with systemic corticosteroids and narrowband UVB. Cutan Ocul Toxicol. 2012;31(1):67-69.

32. Robinson A, Van Voorhees AS, Hsu S, et al. Treatment of pustular psoriasis: from the Medical Board of the National Psoriasis Foundation. J Am Acad Dermatol. 2012;67(2):279-288.

33. Bangale-Daflapurkar S, Danve A. Pustular psoriasis of pregnancy successfully treated with cyclosporine. Am J Ther. 2016;23(5):e1250-e1252.

34. Lakshmi C, Srinivas CR, Paul S, Chitra TV, Kanchanamalai K, Somasundaram LS. Recurrent impetigo herpetiformis with diabetes and hypoalbuminemia successfully treated with cyclosporine, albumin, insulin and metformin. Indian J Dermatol. 2010;55(2):181-184.

35. Patsatsi A, Theodoridis TD, Vavilis D, et al. Cyclosporine in the management of impetigo herpetiformis: a case report and review of the literature. Case Rep Dermatol. 2013;5(1):99-104.

36. Umezawa Y, Ozawa A, Kawasima T, et al. Therapeutic guidelines for the treatment of generalized pustular psoriasis (GPP) based on a proposed classification of disease severity. Arch Dermatol Res. 2003; 295(Suppl 1):S43-S54.

37. Sheth N, Greenblatt DT, Acland K, Barker J, Teixeira F. Generalized pustular psoriasis of pregnancy treated with infliximab. Clin Exp Dermatol. 2009;34(4):521-522.

38. Katz JA, Antoni C, Keenan GF, Smith DE, Jacobs SJ, Lichtenstein GR. Outcome of pregnancy in women receiving infliximab for the treatment of Crohn's disease and rheumatoid arthritis. Am J Gastroenterol. 2004;99(12):2385-2392.

39. Puig L, Barco D, Alomar A. Treatment of psoriasis with anti-TNF drugs during pregnancy: case report and review of the literature. Dermatology. 2010;220(1):71-76. 
40. Adachi A, Komine M, Hirano T, et al. Case of generalized pustular psoriasis exacerbated during pregnancy, successfully treated with infliximab. J Dermatol. 2016;43(12):1439-1440.

41. Saito-Sasaki N, Izu K, Sawada Y, et al. Impetigo herpetiformis complicated with intrauterine growth restriction treated successfully with granulocyte and monocyte apheresis. Acta Derm Venereol. 2017;97(3): $410-411$.

42. Kondo RN, Araújo FM, Pereira AM, Lopes VCH, Martins LMM. Pustular psoriasis of pregnancy (impetigo herpetiformis) - case report. An Bras Dermatol. 2013;88(6 Suppl 1):186-189.

43. Yang CS, Teeple M, Muglia J, Robinson-Bostom L. Inflammatory and glandular skin disease in pregnancy. Clin Dermatol. 2016;34(3): 335-343.
44. Luewan S, Sirichotiyakul S, Tongsong T. Recurrent impetigo herpetiformis successfully treated with methotrexate: a case report. $J$ Obstet Gynaecol Res. 2011;37(6):661-663.

45. Cravo M, Vieira R, Tellechea O, Figueiredo A. Recurrent impetigo herpetiformis successfully treated with methotrexate. J Eur Acad Dermatol Venereol. 2009;23(3):336-337.

46. Lim H-H, Kim S-G, Seo H-G, Kim Y-S, Lee H-J. Recurrent impetigo herpetiformis of pregnancy successfully treated with acitretin. Soonchunhyang Medical Science SMS. 2016;22(1):27-30.
International Journal of Women's Health

\section{Publish your work in this journal}

The International Journal of Women's Health is an international, peerreviewed open-access journal publishing original research, reports, editorials, reviews and commentaries on all aspects of women's healthcare including gynecology, obstetrics, and breast cancer. The manuscript management system is completely online and includes

\section{Dovepress}

a very quick and fair peer-review system, which is all easy to use. Visit http://www.dovepress.com/testimonials.php to read real quotes from published authors.

Submit your manuscript here: http://www.dovepress.com/international-journal-of-womens-health-journal 